\title{
TOWARDS A STRESS CRITERION OF INJURY - AN EXAMPLE IN CAUDOCEPHALAD ACCELERATION*
}

\author{
Y. KING LIU† \\ Department of Engineering Mechanics, The University of Michigan, Ann Arbor, Mich. 48104. U.S.A.
}

\begin{abstract}
The usual specification of acceleration (in number of $g$ 's) as the injury criterion in the biodynamic response of the human body to vibration and/or shock is quite misleading if not invalid. An injury criterion based on stress (or strain) is proposed and shown to have the potential of deciding where, when and how injury is sustained. An example in pilot ejection is used for illustration.
\end{abstract}

\section{INTRODUCTION}

THE SPECIFICATION of the number of $g$ 's allowable as the injury criterion has been in existence for many years. The reasons for its popularity are: (1) acceleration is relatively easy to measure and (2) the concept fits hand in glove with the usual lumped-parameter model employed as the idealization of the biomechanical system.

Consider a pilot forced to eject vertically by catapult. He quickly acquires two motions: (a) a rigid-body motion having a trajectory which will clear him from the aircraft and (b) a 'wave' travels from the seat upward toward the head. Assuming no mishaps during the ejection trajectory, one must now worry whether or not there has been any biomechanical injury due to the 'compression wave'. Roughly speaking, there appears to be a concensus that if the chair acceleration is less than $20 \mathrm{~g}$, the likelihood of injury is not high. These sort of 'go-no go' data have been the basis of design for many years.

\section{EXPERIMENTAL CRTTQUE OF LUMPED-PARAMETER MODEL}

The mathematical idealization of the human body for this case is that of a spring (the spine) articulating a mass (the upper torso). If the chair acceleration is a rectangular pulse, the response is given in terms of the 'overshoot' or dynamic load factor, $D$. $D$ is defined as the ratio of the maximum spring force to the static spring force. For a linear spring, $D \leqslant 2$, while for a nonlinear one it can be larger than two, see Liu and Murray (1966). The essential point to be made here is that the dynamic spring force and the maximum system acceleration are both functions of relative amplitude (for the linear case, they are proportional).

Experimental evidence, Lissner and Evans (1963), using strain gages and/or accelerometers mounted on cadavers show reasonable correlation for motions measured at the crest of the ilium and the sternum. However, the head experienced much higher accelerations than indicated by a simple linear spring-mass model, see Fig. 1. For instance, the dynamic load factor or 'overshoot' in Fig. 1 for the head is $1.63 / 0.63=2.59$, while for the lumbar region it is $0.58 / 0.38=1.53$ (the strain is assumed proportional to the force).

Clinical observations on the condition of the spine of 55 Swedish pilots, who had to eject by catapult from their aircraft, were reported by Hirsch and Nachemson (1961). Thirteen, or about one fourth of the subjects were found to have incurred vertebral fractures. The injuries were invariably compression fractures of the vertebral bodies, especially the endplates. In five cases a single vertebra was

*First received 16 July 1968; in revised form 30 September 1968.

tCurrently NIH Special Research Awardee and Visiting Assistant Professor, Department of Aeronautics and Astronautics, Stanford University 1968-69. 

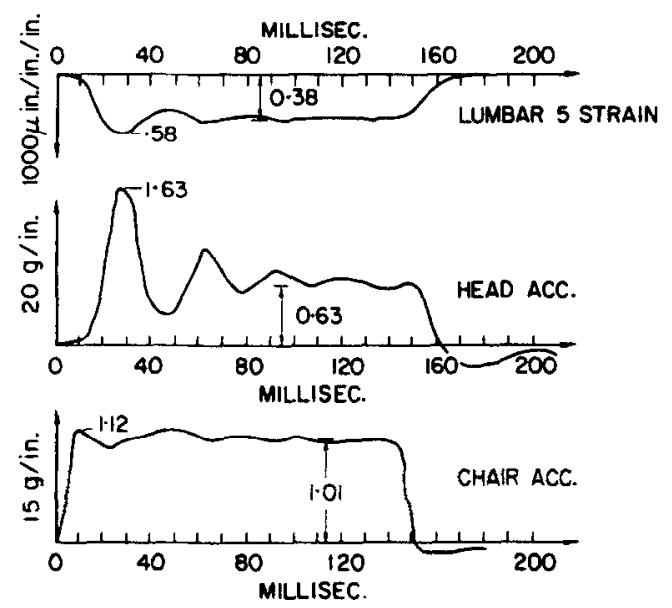

Fig. 1. Typical experimental record (Lissner and Evans, 1963).

damaged whereas eight cases showed multiple fractures. Figure 2 summarizes the incidence, distribution and the level of vertebral fractures that were detected. It is interesting to note that the middle part of the thoracic spine was subjected to the highest frequency of fracture followed next by the lumbar region.

A recent paper by Henzel, Mohr and von Gierke (1968) summarizes and expands on the mechanism of spinal injuries due to pilot ejection together with data on the statical load carrying capacity of many vertebrae. It also indicates the dynamical implications of these results.

No amount of analytic pyrotechnics with the single-degree-of-freedom lumped-para-

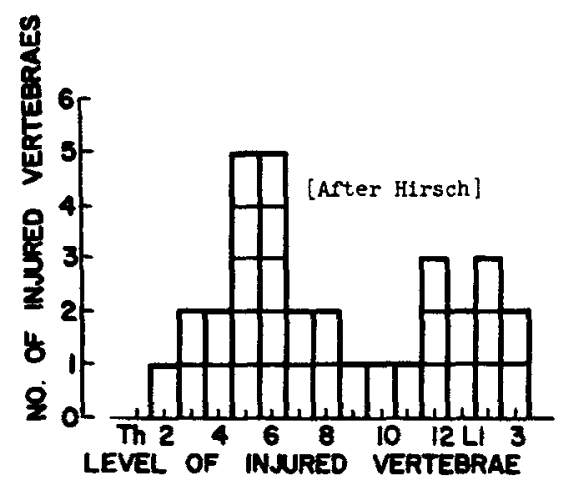

Fig. 2. Incidence, distribution and level of vertebral fracture in pilot ejection (Hirsch and Nachemson, 1961). meter model can possibly explain or fit the experimental data and/or clinical observations quoted above. One can, of course, increase the degrees-of-freedom, such as the seven-mass system proposed by Coermann et al. (1960). However, such a system has three inherent limitations: (a) it has a limited frequency content; (b) a 'wave' is transmitted with infinite speed, i.e., any motion of the first mass is immediately felt by the last mass, which is physically untenable: (c) the analysis of such a system is far from a simple task especially if certain nonlinearities are taken into account.

\section{FIRST-GENERATION CONTINUUM MODELS}

In order to obtain wave responses, a continuous type model is needed. Hess and Lombard (1958) proposed the uniform linear elastic rod as the wave-guide model for the upper torso. By varying the wave speed $c$ of the rod they were able to obtain a 'close' fit to the measured response of the head of test subjects. The time of wave arrival thus determined averaged $25 \mathrm{msec}$ with a range of from 20 to $30 \mathrm{msec}$. A very simple continuum model with an adjustable parameter, time of wave travel, was developed for determining the motion of the head when the vertebral column is subjected to known longitudinal accelerations at its lower end. However, since 'matching' of experimental data and analytic results was obtained at only one point on the rod, namely at the head, such a continuum model cannot be justified as a model for predicting injury to the vertebral column. not at least by virtue of the above study alone.

Carrying the continuum approach further, Liu and Murray (1966) proposed and studied a model consisting of a uniform rod capped at one end by a rigid mass, Fig. 3. The rigid end-mass represents either the head or the head and part of the upper torso (e.g., the combined mass of the thoracic cage, limbs and head) and the rod represents the spinal column. The dimensionless form of the equation of motion is 


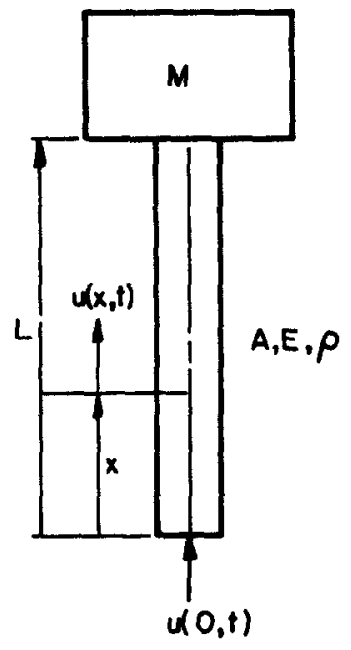

Fig. 3. Continuum model for vertical acceleration.

$$
u_{t t}=u_{x x}
$$

with the initial and boundary conditions as

$$
\begin{aligned}
u(x, 0) & =u_{t}(x, 0)=0 \\
u(0, t) & =f(t) \\
u_{x}(1, t) & =-\lambda u_{t t}(1, t)
\end{aligned}
$$

where subscripts denote partial differentiation. The dimensionless variables are defined as

$$
\begin{aligned}
u=u^{\prime} / l, \quad x=x^{\prime} / l, \quad t=c t^{\prime} / l & \text { and } \\
\lambda & =M / \rho A l,
\end{aligned}
$$

where $x^{\prime}=$ longitudinal coordinate, $u^{\prime}=$ displacement, $\rho=$ mass density, $A=$ crosssectional area, $l=$ length of rod, $t^{\prime}=$ time, $\lambda=$ head to stem mass ratio, $c=$ wave speed $=(E / \rho)^{1 / 2}$ and $E=$ modulus of elasticity.

As a problem in mechanics, the proposed model has been previously investigated by Burr (1950) and Thomson (1960). The former used a recursive mathematical scheme due to Donnell (1930) to compute the effects for several wave-transit times, while the latter made use of the Mellin inversion theorem to obtain an infinite series solution. The Burr solution has the severe restriction that the scheme works only for a step-function input.
Furthermore. the method became unwieldy as the number of wave transits increased beyond three. The Thomson solution turned out to be non-computable because the infinite series is very slowly convergent, if at all. The method detailed in (Liu and Murray, 1966) and summarized below avoids both these difficulties.

Taking the Laplace transform of equations (1)-(4) yields

$\bar{u}(x, p)=$

$\bar{f}(p)\left[\mathrm{e}^{-p x}+\alpha(p) \mathrm{e}^{-p(2-x)}\right]\left[1+\alpha(p) \mathrm{e}^{-2 p}\right]^{-1}$,

where $\alpha(p)=(1-\lambda p) /(1+\lambda p)$ and $p$ is the transformed variable. Application of the binomial, shifting the convolution theorems give the following exact solution for an arbitrary pulse, $f(t)$, i.e.,

$$
\begin{aligned}
u(x, t)= & f(t-x)+ \\
& \sum_{n=1}^{\infty}[f(t-x-2 n)-f(t+x-2 n)] \\
& +\sum_{n=1}^{\infty} \sum_{\nu=1}^{n}(-1)^{\nu} \frac{2^{\nu}}{\lambda^{\nu}(\nu-1) !}\left(\begin{array}{l}
n \\
\nu
\end{array}\right) \\
& \int_{0}^{t}[f(\xi-2 n-x)-f(\xi-2 n+x)] \\
& \times(\xi-t)^{r-1} \mathrm{e}^{-\lambda(\xi-t)} \mathrm{d} \xi,
\end{aligned}
$$

where $f(t)=0$ for $t<0$. The results for a step function acceleration pulse (i.e., corresponding to $\left.\bar{f}(p)=1 / p^{3}\right)$ is displayed in Fig. 4 for several wave transit times. The ordinate is given in terms of dimensionless stress and acceleration. The actual stress, $\sigma^{\prime}(x, t)$, is related to its dimensionless counterpart by

$$
\sigma(x, t)=\sigma^{\prime}(x, t) / E=-u_{x}(x, t) .
$$

If the injury criterion is the compression failure of the vertebral walls, then Fig. 4a shows that injury will occur in the lumbar region after two wave transits - in good agreement with clinical and experimental data; Lissner and Evans, 1963; Hirsch and Nachemson, 1961. 

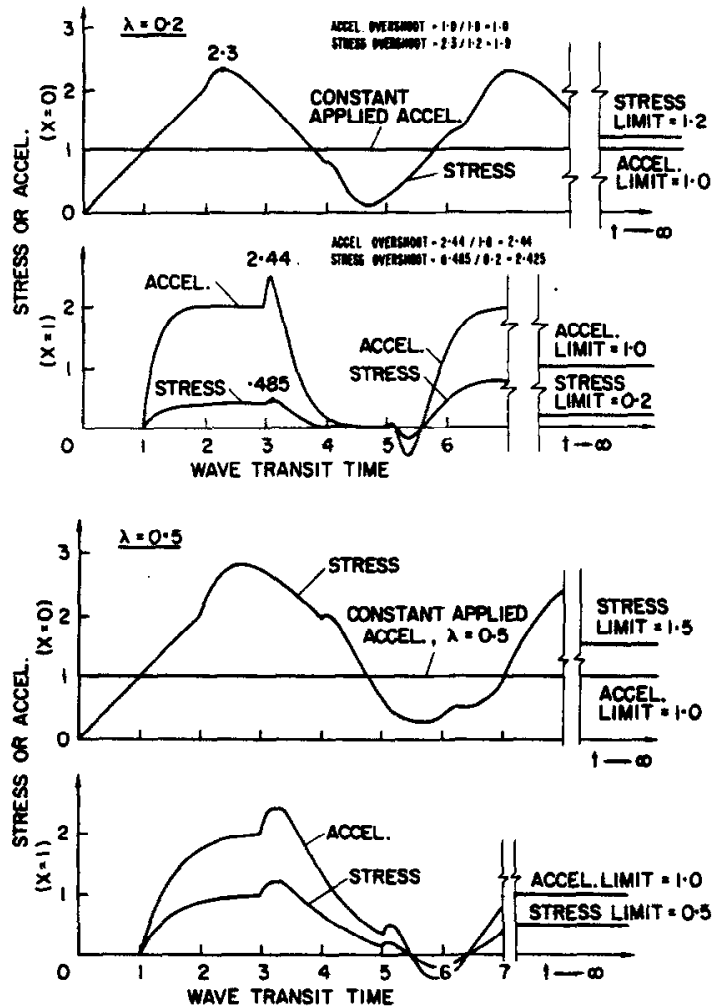

Fig. 4. Theoretical predictions for acceleration and stress: (a) and (b).

\section{LARGE-TIME LIMIT AND THE OVERSHOOT"}

The expression for compressive stress in the transformed variable $p$ is

$\sigma(x, p)=-\bar{u}_{x}(x, p)=$

$-\bar{f}(p)\left[p \mathrm{e}^{-p x}+\alpha(p) \mathrm{e}^{-\alpha(2-x)} p\right]\left[1+\alpha(p) \mathrm{e}^{-2 p}\right]^{-1}$.

The large time limit is found from the Tauberian or Final-value theorem which states: If $\sigma(x, t)$ and $\partial \sigma(x, t) / \partial t$ are Laplace transformable and the limit of $\sigma(x, t)$ exists, then the behavior of $\sigma(x, t)$ in the neighborhood of $t \rightarrow \infty$ corresponds to the behavior of $-p \bar{u}_{x}(x, p)$ in the neighborhood of $p \rightarrow 0$, (see Thomson, 1960). Proceeding formally for $\bar{f}(p)=1 / p^{3}$, i.e., a step acceleration input, one encounters the $0 / 0$ indeterminate form. Using L'Hospital's rule once will yield readily

$$
\lim _{t \rightarrow \infty}[\sigma(x, t)]=1+\lambda-x .
$$

We now examine whether the conditions on $\sigma(x, t)$ are satisfied. Both $\sigma$ and $\partial \sigma / \partial t$ are Laplace transformable, but the limit of $\sigma(x, t)$ does not exist since the system has no dissipation. What then is the meaning of the limit shown in (10)? It is precisely the stress on the bar due to the dead weight above the coordinate under consideration, e.g., $\sigma(1, t) \rightarrow$ $\lambda$ and $\sigma(0, t) \rightarrow 1+\lambda$. These statical stress values are the asymptotes to which the responses tend to if dissipation were included. A similar situation exists for lumped-parameter damped and undamped systems. In view of (4) and (10), one gets

$$
\lim _{t \rightarrow \infty}\left[u_{t t}(1, t)\right]=1 \text {. }
$$

The 'overshoot' is now defined as the ratio of the maximum dynamical value (of acceleration, stress or strain) to its statical equilibrium value. Since stress and acceleration are different things, it is interesting to compare the overshoot using these quantities. For instance, using $\lambda=0.2$, we get from Fig. 4a:

(a) At $x=0$ (load end)

Acceleration overshoot $=1 \cdot 0 / 1 \cdot 0=1 \cdot 0$

Maximum dimensionless stress $=2 \cdot 3$

Stress overshoot $=2 \cdot 3 / 1 \cdot 2=1 \cdot 9$.

(b) At $x=1$ (head end)

Acceleration overshoot $=2 \cdot 44 / 1 \cdot 0=2 \cdot 44$

Maximum dimensionless stress $=\mathbf{0 . 4 8 5}$

Stress overshoot $=0.485 / 0.2=2.425$.

It is significant to note that low overshoots correspond to high absolute stress values $(x=0)$ and vice versa.

The above examples point out clearly that the absolute value of stress is the meaningful injury criterion and the 'overshoot' concept is, in fact, quite misleading, if not invalid.

\section{DISCUSSION OF RESULTS AND GENIRALIZATIONS}

It might seem far-fetched to model the spine by a rod. Its justification was most succinctly stated by Payne (1961) for lumped-parameter models: "For short-duration accelerations, however, the soft, low-frequency parts of the 
body (such as the viscera) do not deflect far enough to load up the spine,...". These remarks when applied to the present model means that the model is valid only for shortduration accelerations. If waveshape correspondence is to be better than indicated by comparing Figs. 1 and 4, one must incorporate the many factors which have not been taken into account, e.g., damping, internal (muscles, viscera) and external (seat belts) constraints, curvature, nonuniformity of mass distribution and section properties, nonlinear material properties, etc., etc.

Damping can be incorporated into the model through the one-dimensional 'correspondence principle' in viscoelasticity (Flügge, 1967). It can be shown that the maximum and equilibrium values are only slightly affected by the viscoelastic properties, but the waveshape of the response is altered.

By considering the upper torso as a onedimensional but complex bar-type structure, it is possible to take into account the transmission and refiection of stress waves through elements with gradual changes and discontinuities in area and material as well as the presence of rigidly attached masses. The technique is essentially a modification of the well-known method of characteristics (Kolsky, 1963).

The above refinements are in progress but they do not detract from the observations that the spatial distribution of the human body is of primary importance. In fact, its consideration is the first step towards an understanding of where, when and how injuries occur.

Acknowledgement - This investigation was supported in part by Public Health Service Research Grant No. UI-00025-02 from the National Center for Urban and Industrial Health.

\section{REFERENCES}

Burr, A. H. (1950) Longitudinal and torsional impact in a uniform bar with a rigid body at one end. J. appl. Mech. 17, 209-217.

Coermann, R. R., Ziegenruecker, G. H., Wittwer, A. L. and von Gierke, H. E. (1960) The passive dynamic mechanical properties of the human thorax-abdomen system and of the whole body system. Aerospace $M$ ed. 31, 443-455.

Donnell, L. H. (1930) Longitudinal wave transmission and impact. Trans. ASME 52, 153-167.

Flïge, W. (1967) Viscoelasticity. Blaisdell.

Henzel, J. H., Mohr, G. C. and von Gierke, H. E. (1968) Reappraisal of biodynamic implications of human ejections. Aerospace Med. 39, 231-240.

Hess, J. L. and Lombard, C. F. (1958) Theoretical investigations of dynamic response of man to high vertical accelerations. J. Aviat. Med. 29, 66-74.

Hirsch, C. and Nachemson, A. (1961) Clinical observations on the spine in ejected pilots. Acta orthop. scand. 31, 135-145.

Kolsky, H. (1963) Stress Waves in Solids. Dover, New York.

Lissner, H. R. and Evans, F. G. (1963) Effects of acceleration on the human skeleton. Prog. Rpt. to Public Health Service for Grant No. Ac-00054-06.

Liu, Y. K. and Murray, J. D. (1966) A theoretical study of the effect of impulse on the human torso. Biomechanics, Symp., ASME Publ. pp. 167-186.

Payne, P. R. (1961) The dynamics of human restraint systems. Nat. Acad. Sci. Symp. on Impact Acceleration Stress. Brooks AFB, San Antonio, Texas.

Thomson, W. T. (1960) Laplace Transformation. 2nd Edn. Prentice-Hall, New York. 